\section{$\underset{\substack{\text { hommes } \\ \text { \& migrations }}}{ }$}

\section{Hommes \& migrations}

Revue française de référence sur les dynamiques

migratoires

\section{$1297 \mid 2012$}

Migrations en création

\title{
Ken Bugul
}

Les chemins d'une identité narrative

\section{Nicolas Treiber}

\section{Q OpenEdition}

1 Journals

\section{Édition électronique}

URL : http://journals.openedition.org/hommesmigrations/1545

DOI : 10.4000/hommesmigrations. 1545

ISSN : 2262-3353

Éditeur

Musée national de l'histoire de l'immigration

\section{Édition imprimée}

Date de publication : 1 mai 2012

Pagination : 44-55

ISSN : 1142-852X

\section{Référence électronique}

Nicolas Treiber, « Ken Bugul », Hommes \& migrations [En ligne], 1297 | 2012, mis en ligne le 31

décembre 2014, consulté le 01 mai 2019. URL : http://journals.openedition.org/

hommesmigrations/1545; DOI : 10.4000/hommesmigrations.1545 


\section{Ken Bugul Les chemins d'une identité narrative}

Par Nicolas Treiber, doctorant en littérature française et comparée, université Sorbonne-Paris-IV

La rencontre de l'autre comme matière littéraire. C'est l'objet de la trilogie autobiographique de Ken Bugul, auteur sénégalaise, qui explore les tréfonds de l'identité humaine malmenée par l'histoire. Un parcours de la non-reconnaissance mis en scène entre le Sénégal, la Belgique et la France qui débouche sur l'abîme de la perte de repères

identitaires, ontologiques. Mais Ken Bugul n'en reste pas là. L'unité en devenir de sa personne trouvera son point d'appui dans cette œuvre qui jalonne le chemin de l'apaisement. 
Certains pensent qu'ils font un voyage, en fait, c'est le voyage qui vous fait ou vous défait.

Nicolas Bouvier, L'Usage du monde

"Ken Bugul" est un nom pour conjurer le sort. On le donne au Sénégal à un nouveauné dont la mère a fait plusieurs fausses couches. "Ken Bugul" signifie en wolof "celle dont personne ne veut, même pas la mort. Ken Bugul est le nom de plume d'une écrivaine née en 1947 au Sénégal, auteure d'une ceuvre sensible, intime, au carrefour du témoignage et de la fiction. Un prénom de rescapée comme nom d'auteur. De la demi-douzaine de livres écrits par Ken Bugul, un ensemble se dégage, formé par trois livres qui content quelques épisodes d'une vie éclatée, hachurée, qui finalement parvient à l'unité. Ken Bugul est à la fois l'auteure, la narratrice et le personnage de ces récits. Dans Le Baobab fou, Cendres et Braises puis Riwan ou le chemin de sable, elle met en scène une trajectoire qui conduit la narratrice âgée de 20 ans du Saloum en Belgique, puis à Paris vers la trentaine. Au départ, un voyage pour études ; à l'arrivée, une chute dans la spirale de l'errance, de l'oubli de soi, et de la perte des repères affectifs et idéologiques.

D'où écrit Ken Bugul ? Ses récits au passé semblent l'indiquer : depuis le lieu de l'apaisement, depuis l'espace euphorique de la paix retrouvée où s'enchâsse la chronique de sa déréliction. Ces trois livres de Ken Bugul ne narrent rien d'autre au fond qu'un itinéraire chaotique mais déterminé vers soi-même, par-delà son intégrité menacée par ses rencontres, les fissures de sa vie, les ornières de l'Histoire. En jeu, l'unité d'une personne, sa possibilité d'exister soi-même parmi les siens, son pouvoir de l'exprimer, de se dire. Une possibilité conquise sur le fil de l'existence même. L'aventure s'est bien terminée mais elle a été violente: le passage par l'école coloniale, l'assimilation d'autres références culturelles que les siennes propres, le sentiment de ne plus trouver sa place, d'être un être déraciné qui va puiser dans le mirage de l'ailleurs des racines fantasmées, et, là-bas, l'épreuve du regard de l'autre, sans pitié. Au fil de ces trois romans, Ken Bugul détaille par le menu le contenu de l'expérience migratoire, les effets du voyage sur une personne qui ne s'est jamais vraiment sentie nulle part chez elle.

La matière même de son acte d'écriture est la mise en scène d'une identité narrative, c'est-à-dire de la structure de son expérience de vie. Ses romans en dévoilent les ressorts, les zones d'ombre et la cohérence problématique. Parce que la littérature éclaire les rouages du vécu, et confère de la lisibilité à des histoires de vie, il importe de saisir dans l'expérience de Ken Bugul telle qu'elle nous est racontée les lignes de force qui 
déterminent l'unité et le devenir d'une personne qui fait l'épreuve de l'ailleurs, qui traverse les espaces tête baissée et donne à son voyage des airs de fuite en avant. "Il n'y a pas de récit éthiquement neutre. La littérature est un vaste laboratoire où sont essayés des estimations, des évaluations, des jugements d'approbation et de condamnation par quoi la narrativité sert de propédeutique à l'éthique ${ }^{(1)}$." En suivant le lien que Paul Ricceur établit dans Soi-même comme un autre entre identité narrative et questionnement éthique, on peut s'interroger sur les enjeux éthiques et ontologiques que posent les livres de Ken Bugul : Qu'est-ce qui fonde la permanence d'une personne partagée entre deux mondes? Sur quoi repose sa capacité d'action et de réaction face aux aléas de son existence, son pouvoir de création d'un rapport apaisé à l'autre?

\section{De nouveaux repères sur une absence de repères}

L'enfance de Ken Bugul s'est déroulée durant la colonisation. L'école coloniale est à l'origine, indirectement, d'une déchirure fondatrice dans son existence. "J'étais allée à l'école française, la plus jeune de l'unique classe et la seule fille de ma famille à compter toutes les générations, à avoir franchi le seuil d'une école [...]. L'école française qui allait bouleverser mille mondes et mille croyances qui se cachaient derrière les baobabs médusés en prenant des formes humaines ${ }^{(2)}$." Cette entrée à l'école a provoqué un arrachement ancien mais qui conserve une profondeur abyssale et dont le souvenir irradie Le Baobab fou et Cendres et Braises : l'abandon momentané, mais vécu comme définitif, de la mère séparée du père, et l'incompréhension qu'il a suscitée chez Ken Bugul alors âgée de 7 ans. "Je reprochais à la mère de ne pas m'avoir emmenée avec elle [...].Je n'avais pas toujours su pourquoi la mère avait dî partir. - Mais tu allais à l'école, c'est pour cela que je ne t'ai pas emmenée avec mo $\imath^{(3)}$." Cet événement est la cause du long chemin sinueux que Ken Bugul a dû entreprendre pour passer outre, avancer malgré tout, se construire audessus d'une faille : la perte du lien avec la mère.

Or ce lien perdu a été remplacé par d'autres que l'auteur détisse au fil des pages. L'entrée à l'école a signé pour Ken Bugul la coupure vis-à-vis de ses proches, de sa société. Elle s'identifie à la nouvelle culture. "Tout ce qui m’intéressait à l'époque, c'était l'école française. Je m'étais rendu compte que javais une facilité d'assimilation qui me permettait d'apprendre et il y avait beaucoup à apprendre et il y en avait pour tout le monde ${ }^{(4)}$." Hormis l'événement biographique évoqué, un processus d'aliénation s'est irrémédiablement mis en marche. Et c'est lui qui conditionne rétrospectivement son départ vers la Belgique pour poursuivre ses études grâce à une bourse de l'Office de la coopération au développement : "Ce matin-là, nous nous faisions nos adieux. Je partais. 
Les autres restaient. Je partais très loin. Je m'arrachais pour tendre vers le Nord. Le Nord des rêves, le Nord des illusions, le Nord des allusions. Le Nord référentiel, le Nord Terre promise ${ }^{(5)}$." L'école et le mode de vie occidentaux importés durant la période coloniale, en inculquant de nouvelles références culturelles, introduisent des césures dans l'espace de départ, et l'Indépendance n'y change rien. La polarisation vers le "Nord référentiel" a pour contrepoint la dépréciation de l'espace d'origine, de son fonctionnement : "Durant ces premières années d'indépendance, je ne songeais qu'à mon émancipation. Je voulais être une femme bardée de diplômes qui épouserait un homme bardé de diplômes de l'école occidentale. [...] À l'école on m'avait appris à considérer les hommes de mon village comme des sauvages, des gens qui ne connaissaient pas les bonnes manières, faisaient l'amour avec brutalité, ne respectaient pas la femme et s'accouplaient à tort et à travers ${ }^{(6)}$." Ken Bugul traduit l'intériorisation d'un système de représentations et de valeurs qui disqualifie celui de sa propre société. C'est bien là la marque d'une aliénation culturelle, d'une colonisation des esprits dont Ken Bugul fera les frais là-bas, en Belgique dans Le Baobab fou, à Paris dans Cendres et Braises, quand le regard exotisant des autres, des hommes en particulier, la renverra sans cesse au miroir de sa peau et de son origine. Bercée d'illusions, Ken Bugul se trouve, dès le départ, dans la situation critique d'"une femme noire, une étrangère, plus fragile encore $e^{(7) "}$. Pour comprendre l'impact de la situation coloniale, sa rémanence et son pouvoir de nuisance dans son premier voyage, qu'elle fait pourtant quelques années après les indépendances, on ne saurait trop insister sur une conséquence particulière de l'entreprise coloniale. Frantz Fanon a bien relevé l'aporie de la situation coloniale en matière culturelle. Un pouvoir de déculturation qui détermine pour une grande part les réactions du colonisé face à la culture occidentale et face à la sienne

La polarisation vers le "Nord référentiel" a pour contrepoint la dépréciation de l'espace d'origine, de son fonctionnement. propre. "Pour l'Africain la société blanche a brisé son ancien monde sans lui en donner un nouveau. [... Elle barre la route de l'avenir après avoir fermé la porte du passé( ${ }^{(8)}$." En effet, la relation coloniale repose sur une dépossession, une expropriation fondamentale, chez le colonisé, de sa capacité d'action : "Le bouleversement a atteint le Noir de l'extérieur. Le Noir a été agi $\imath^{(9)}$." Dans les romans de Ken Bugul, cette déréliction culturelle sert de terreau à l'aventure de la narratrice. Son voyage d'études est irrémédiablement marqué par le sceau de cette passivité. Elle est davantage voyagée qu'elle ne voyage vraiment, tant ce qu'elle découvre ne correspond que trop rarement à ses attentes, tant elle est ballottée par les événements, à la merci de forces qui la dépassent. Au fil de son parcours erratique, l'enjeu se précise : la reprise en main de sa propre histoire. 


\section{Non-réciprocité et instrumentalisation}

Au seuil du départ, comme beaucoup d'autres colonisés acculturés, Ken Bugul se trouve enlisée dans un rapport de domination qui s'appuie sur la diffusion d'une sous-culture. Selon Bernard Mouralis : "Le choix, s'il existe, ne peut être qu'entre une caricature de culture européenne et ce que le colonisateur a laissé subsister de la culture autochtone ${ }^{(10)}$."Ce rapport de domination culturelle a ceci de particulier qu'il est fondé sur la non-réciprocité. Il se nourrit des attentes qu'il sait produire sans jamais les satisfaire. C'est ici que Bernard Mouralis identifie le point de naissance de l'individu "déraciné". "Les intellectuels ne pensent pas souvent que ce qu'ils considèrent comme leurs acquis au contact du colonisateur ne leur est pas reconnu par celui-cili1."

Cette relation clivée entre des attentes et un monde où elles ne sont pas réciproques se cristallise chez Ken Bugul dans la manière dont le regard de l'autre la construit. "J'étais souvent avec les Blancs ; je discutais mieux avec eux, je comprenais leur langage. Pendant vingt ans je n'avais appris que leurs pensées et leurs émotions. Je pensais m'amuser avec eux, mais en fait jétais plus frustrée encore : je m'identifiais en eux, ils ne s'identifiaient pas en mo ${ }^{\{12)}$." Ken Bugul abandonne ses études, fréquente les milieux aisés et les artistes, joue à fond le “jeu de l'Occident”. Pourtant, elle est acceptée sans être comprise dans sa quête identitaire, juste pour la touche de couleur qu'elle apporte dans les soirées mondaines d'une société qui s'exotise et se complaît dans l'autosatisfaction de sa tolérance feinte. "J'allais donc partout et j'étais partout celle qu'on remarquait. Parce qu'elle était noire, et aussi parce que, par désespoir, elle s'accrochait et elle osait [...]. Ces gens riches étaient libres de faire ce qu'ils voulaient, ils absorbaient la diaspora pour l'originalité. 'Nous avons une amie noire, une Africaine', était la phrase la plus 'in' dans ces milieux ${ }^{(13) . "}$ "J'étais cette négresse, cette 'chez vous autres', cette 'toi, en tant que noire, il faudrait que..., cet être supplémentaire, inutile, déplacé, incohérent ${ }^{(14)}$."

Parce qu'elle est en manque de repères affectifs, enfermée en terre étrangère dans une relation non réciproque, avec l'autre, Ken Bugul fait le pari de la provocation. "J'étais une Noire, provocante, sophistiquée, qui connaissait leur culture, leur civilisation. Ils en étaient surpris ${ }^{(15)}$." Ce faisant, elle se fait réifier, chosifier, par un regard qui la cantonne à une altérité radicale où l'exotisme voisine avec une vision de genre, où le racisme latent se joint au machisme pour lui renvoyer le reflet de ses égarements et s'en repaître. "J'essayais de scandaliser la société, dans des robes transparentes aux couleurs vexantes [...] le jeu de la couleur noire : être une femme noire qui plaise à l'homme blanc [...]. Moi qui avais rêvé d'un foyer, d'un père, d'une mère, d'ancêtres, moi qui voulais être reconnue! J'étais jetée dans la cage des fantasmes inassouvis et des chevauchées dans le rêve surréel ${ }^{(16)}$." 
Dans ce contexte, sa quête éperdue de reconnaissance et sa grande vulnérabilité ont deux conséquences. D'une part, elle ne parvient pas à entretenir de liens avec les autres Africains : "Je n'arrivais pas à redémarrer. [...] Le temps passait en introspections, en quête de racines imaginaires. [...] Je n'arrivais pas à me lier avec les Africains des autres nationalités. Les envahisseurs nous avaient séparés, portés les uns contre les autres et nous n'étions pas arrivés à nous en sortir rit.' D'autre part, elle devient le jouet de son apparence. Exilée à la surface d'elle-même, commence le glissement fatal vers ces régions des relations humaines où la personne bafouée risque sa vie.

\section{La mécanique du glissement}

Dans Le Baobab fou, c'est l'expérience des bars interlopes qui va faire office de déclencheur. Alors qu'elle plonge de plus en plus dans une introspection sans issue au point de se dissoudre dans le flux des événements - "Et je me réfugiais dans ce système : faire de la vie un aléa inconscient : deux tours de ronde avec la disponibilité(18)" - elle découvre les salons de massage, se fait embaucher comme danseuse dans un bar. Mais Ken Bugul fait une piètre entraîneuse. Ce qu'elle cherche c'est l'humanité, l'échange. Et c'est tout le contraire qu'on lui propose : "Tu plais aux hommes. Ken, tu es une Noire, tu peux te faire une fortune ${ }^{(19)}$." La rencontre d'une Suissesse et de deux Tunisiens lui entrouvre le monde de la prostitution. Le corps, la couleur pour unique consistance, le personnage perd son épaisseur et, très vite, cette néantisation va déboucher sur le retour en Afrique. "J'avais pris l'avion folle de rage et de désespoir. Le non-retour des choses avait amputé la conscience. Le rétablissement était devenu impossible. Rétablissement de l'enfance perdue, envolée un après-midi, la première fois que j'avais vu un Blanc (20)." Mais ce retour s'avère lui aussi être une impasse, c'est le sommet tragique du Baobab fou. Ken Bugul est toujours en recherche du lien familial, de son contenu, des racines de son être. Elle a manqué le rendez-vous avec elle-même, ses retrouvailles, comme le lui signale l'ombre morte du baobab qui l'a vue grandir puis partir. "Sans paroles, je prononçais l'oraison funèbre de ce baobab témoin du départ de la mère, le premier matin d'une aube sans crépuscule. Longtemps, je restais là devant ce tronc mort, sans pensée $e^{211}$."

Dans Cendres et Braises, la mécanique de déréliction est la même, à ceci près que la narration s'effectue depuis l'espace du retour, par analepse. Les mésaventures qu'elle a connues, cette fois en France, avec un homme violent et jaloux sont enchâssées dans le récit de sa reconstruction. Plutôt que de "mésaventures" il vaudrait mieux parler de la spirale infernale de la violence, celle où l'amour confine à la haine et produit autant de femmes humiliées et battues que d'hommes tyranniques et inhumains. Dans ce livre, Ken Bugul conte le deuil d'un amour aussi fort que dévastateur, avec un 
homme d'affaires plus âgé qu'elle a rencontré au Sénégal après son retour de Belgique. Venue en France, elle découvre qu'il est marié et endosse l'habit de la maîtresse cachée - pas pour longtemps -, vouée à son homme et dépendante de lui.

Plus que jamais, discrimination de genre et domination culturelle se combinent dans l'aliénation: "Y. me voulait dans sa culture, intégralement ${ }^{22) " ; ~ " L a ~ r e ́ a l i t e ́ ~ a f r i c a i n e, ~ j e ~ n e ~}$ la vivais pas (23)"; "J'aimais cet homme à tel point que je n'avais plus que lui; avec le reste du monde, le fossé était immense(24)." Que de forces ne faut-il pas pour sortir de cette situation, cautériser la coupure avec les autres et briser la relation de dépendance avec un homme qui déverse sa rancceur et ses frustrations à coups de poing. " Cet homme qui disait m'aimer, qui m'avait aimée, qui disait oublier que jétais noire tellement jétais assimilée à lui, comment pouvait-il en me traitant de sale négresse essayer de m'humilier, de me rabaisser en tant qu'être humain ${ }^{(25)}$ ?" Dans cette "valse diabolique" entre une femme noire et un homme blanc, Ken Bugul excelle à mettre en scène les dérapages de l'intimité, la difficile séparation, l'oraison funèbre d'un amour monstre.

\section{Une identité en attente de réponses}

Cendres et Braises a un statut particulier par rapport aux deux autres livres étudiés : il fait office de pivot, il se tient entre deux espaces, il dévoile des abîmes déjà comblés. Le récit est pris en charge par une narratrice rentrée auprès de sa mère au village, après être passée par la ville où elle n'était pas parvenue à se retrouver : "Le retour récupérait mal. [...] La ville me récupérait mal quand j'étais arrivée ${ }^{(26)}$." De manière lancinante, son histoire avec Y. passe mal, tout la renvoie à son vécu antérieur. Une nouvelle fois, comme dans Le Baobab fou, Ken Bugul est rentrée vidée de son voyage, anéantie. L'enjeu du retour est bien vital : il s'agit pour elle de mourir ou de renaître. Et ce sera la vie, grâce à la rencontre de "quelqu'un qui allait constituer un élément important au point où jen étais ${ }^{(27) "}$.

Un point commun à toutes les expériences de Ken Bugul, qu'elles soient dramatiques ou euphoriques, est sa capacité à se donner tout entière à ce qu'elle est en train de vivre, parce que rien ne la retient si ce n'est le lien qu'elle cherche désespérément à retisser avec la mère, avec sa terre natale. "Rupture. Non. Pas rupture. Absence/Silence ${ }^{(28)}$." Habituée aux incises poétiques dans sa narration, Ken Bugul formule ainsi ce qui est peut-être la clé de son vouloir être et de sa liberté : "Enracinement. Ouverture. Pas ouverture, plus exactement disponibilité(29)." La volonté de s'appuyer sur des racines et en même temps de s'ouvrir au monde, aux autres, s'affiche clairement. Qu'en est-il de la disponibilité ? S'agit-il de la disponibilité d'esprit de quelqu'un de suffisamment libre pour être réceptif aux sollicitations du monde? Ou, sur un mode plus désespéré, 
de la situation de quelqu'un qui, n'ayant plus rien, est prêt à tout ? "C'était dans ce village que je revenais après des années et des années à l'étranger. [...] Revenir à la Mère, revenir aux origines, revenir aux sources des choses, revenir dans l'environnement, revenir dans l'atmosphère, revenir au familier, revenir pour la confrontation ${ }^{(30)}$." Dans le retour couvent la violence de l'abandon maternel et le ressentiment. Rien n'est réglé, Ken Bugul n'est pas encore arrivée. Le regard des autres leur fait payer, à elle et à sa mère, son absence et ses égarements : "Par les mouvements négatifs de la tête de Maty War, je pensais à tout ce que la Mère avait enduré et endurait sûrement encore à cause de moi. 'Elle n'est pas mariée? Non. Elle a des enfants? Non. Elle travaille? Non. Elle est saine d'esprit? $N_{o n}{ }^{(31)}$." Cette "espèce de folie" dont la charge la rumeur est à prendre au sérieux comme la traduction d'une véritable crise d'identité.

Ken Bugul ne sait pas plus que les habitants de son village où elle habite. Ce qui se joue dans cette crise c'est la permanence de son identité. Ses différents voyages ont constitué l'itinéraire qui l'a conduite à la perte d'elle-même. Ce qu'elle a perdu en chemin c'est la possibilité de se maintenir elle-même parmi les siens. C'est une des dimensions de l'identité que Paul Ricceur désigne dans Soi-même comme un autre sous le terme d' ipséité". Il ne s'agit pas des traits de caractère dont la permanence définit la singularité d'un être, c'est-à-dire sa "mêmeté", mais plus profondément de la dynamique du maintien de soi comme personne. Car Ken Bugul s'est égarée en cours de route et les villageois en sont témoins. "Hé! toi, vraiment tu es perdue, hé ! Tu es restée trop longtemps, tout le monde pensait que tu n'allais plus revenir ${ }^{(32), ", ~ l u i ~ d i t ~ A n t a ~ S e ̀ y e, ~ s o n ~}$ interlocutrice dans le roman. Il s'agit pour Ken Bugul de pouvoir répondre : "Me voici !", attester de sa présence pleine et entière au monde, dans l'univers des origines. Commence un long voyage immobile qui se poursuit dans Riwan ou le chemin de sable, à l'issue duquel elle va trouver une place au sein de sa société traditionnelle.

\section{La disponibilité, ferment de la réconciliation}

En revenant au village, au seuil d'une nouvelle étape de sa vie, Ken Bugul semble remplir une promesse qu'elle s'est faite et qu'elle fait aux autres. C'est l'enjeu de son retour: "Il fallait aussi que je réserve aux miens une partie de ma vie, que nous en vivions ensemble ne serait-ce qu'une portion, mais c'était nécessaire si je voulais mourir chez moi ${ }^{(33)}$." Chez Paul Ricceur, l'identité au sens du maintien de soi repose sur la tenue de la promesse, dont la particularité est d'imposer un défi au temps : "Quand même mon désir changerait, quand même je changerais d'opinion, 'je maintiendrai (34)." Pour réussir son retour, Ken Bugul a dû relever un tel défi. Car le constat est amer et sans appel : 
"Comme je regrettais d'avoir voulu être autre chose, une personne quasi irréelle, absente de ses origines, d'avoir été entraînée, influencée, trompée, d'avoir joué le numéro de la femme émancipée, soi-disant moderne, d'avoir voulu y croire, d'être passée à côté des choses, d'avoir raté une vie, peut-être. Parce qu'on m'avait dit de renoncer àce que jétais alors que jaurais dû rester moi-même et mieux m'ouvrir à la modernitée ${ }^{(35)}$." À la honte, Ken Bugul répond par la promesse, une promesse de fidélité à ses origines. C'est moins la permanence de son caractère qui est en jeu que la tenue de sa parole. Et c'est ici que Paul Ricceur permet d'introduire la disponibilité comme l'attitude de celui qui tient ses promesses vis-à-vis d'autrui, à la croisée de l'ontologie et de l'éthique, quand le maintien de soi, dépendant de la relation à l'autre, est ramené à son socle intersubjectif. "C'est à l'autre que je veux être fidèle. À cette fidélité, Gabriel Marcel donne le beau nom de disponibilité(36)."

La lecture qu'offre Paul Riccur de ce philosophe chrétien peut permettre d'éclairer ce que recouvre la disponibilité chez Ken Bugul. Avec Gabriel Marcel, Paul Ricceur distingue deux dimensions, deux modalités de la disponibilité : "Il y a, d'un côté, une disponibilité selon l'avoir : au sens précis où avoir, c'est disposer de..., c'est-à-dire à la limite pouvoir se débarrasser de... comme il arrive dans le suicide. Il y a, de l'autre côté, une disponibilité selon l'être ; au contraire de l'usage précédent du terme, cette disponibilité consiste à ne pas pouvoir disposer de..., afin de rester disponible pour (l'avenir, les autres, la grâce, Dieu... ${ }^{377}$." On voit comment, d'une part, la métaphore bancaire de l'avoir disponible traduit le pouvoir d'action de la personne qui se possède, qui s'appartient suffisamment pour fonder sa liberté de suivre comme bon lui semble le cours de son existence. C'est une affirmation de l'amour-propre dont la manifestation radicale serait le suicide. Par désespoir, Ken Bugul l'envisage à plusieurs reprises, comme à la fin du Baobab fou : "Mourir. Mourir maintenant. Le suicide pur ! [...] La conscience de tout ce qui métait arrivé si loin du village où je suis née, me faisait prier Dieu de me faire renaître, comme si presque un quart de siècle de tourment n'avait jamais $e^{\prime} e^{\prime(38)}$." Mais Ken Bugul est pugnace dans sa recherche d'une assise à partir de laquelle pouvoir se (re)constuire. Sa volonté de renaissance l'emporte sur la détresse de ne plus s'appartenir.

D'autre part, vue sous l'angle de l'être et non plus de l'avoir, la disponibilité s'appuie sur un profond dénuement qui fait écho à celui de Ken Bugul. Mieux, son parcours littéraire met en scène la transformation de ce vide en don. Ceci peut permettre de prendre la mesure du retour de la narratrice : la disponibilité totale de son être en quête d'unité qui, dans le même temps, s'emploie à digérer la partie occidentale de sa vie, lui permet de se donner aux siens, de leur réserver ce nouveau seuil de son existence qu'elle est en train de franchir avec eux. Et c'est ici qu'intervient le moteur de sa réunification qui puise aux racines de sa société : la rencontre avec le Marabout 
dans Cendres et Braises, le Serigne dans Riwan ou le chemin de sable. Ce chef spirituel musulman d'obédience mouride, affable et curieux de tout, avec qui elle construit des rapports privilégiés, va la prendre pour épouse. "La disponibilité que javais pour recommencer, pour apprendre, pour être, pour vivre, m'avait aussi permis d'accepter que tout à coup on m'annonçât que jétais mariée à un homme à mon insu, un homme qui était un Serigne, un grand Serigne, un homme qui fut avant tout un ami et un confident ${ }^{(39)}$." On aurait tort de lire cette disponibilité chez Ken Bugul comme une faiblesse de la volonté, l'expression d'un anéantissement confinant à la passivité chez celle qui, revenue déçue par les promesses non tenues de la société occidentale, n’a plus le choix. C'est le contraire.

Disponible parce que vidée par ses expériences et à la fois pleine d'une volonté inextinguible de vie, elle ne s'attendait pas à trouver parmi les siens un appui ferme pour sa reconstruction, alors que l'abandon de la mère l'avait depuis lors placée sur la brèche, en partance pour la quête d'un ailleurs fantasmé. "J'avais sous-estimé la capacité des sources, des origines à récupérer les siens. J'avais retrouvé mon village, mes sens, mon milieu, mon moi-même posé dans un petit coin et qui m'attendait depuis. J'étais réintégrée dans la société et remplissais mes engagements vis-à-vis d'elle avec beaucoup de bonheur.Je ne me sentais plus isolée. Je fonctionnais dans un milieu familier, avec les repères de mon environnement et les références de mon éducation traditionnelle ${ }^{(40)}$."

C'est donc avec surprise qu'elle se met en mesure d'accepter sa réinscription, grâce au mariage avec le Marabout, dans l'ordre traditionnel de sa société natale. Cette dernière puise sa forte cohésion aux sources du

\section{Le parcours de Ken Bugul pourrait bien se lire comme la promesse tenue de la réconciliation qui seule peut assurer la permanence d'une identité morcelée par les clivages de l'histoire, les blessures de la vie et la violence du voyage entre deux mondes.} mouridisme dont le ressort est le Ndigueul, 1'“ordre" donné par le marabout, auquel le disciple doit se soumettre. Or Ken Bugul insiste sur le fait que cette soumission n'est rendue possible que par la liberté du disciple qui choisit son marabout et noue avec lui son destin. "On ne suivait pas le Ndigueul à l'aveuglette ni par endoctrinement, ni d'une façon institutionnelle. Le disciple luimême choisissait son Serigne indépendamment de son père ou de sa mère. Ensuite, il prenait son temps pour se préparer à l'idée de se soumettre à ce choix, de se convaincre de son propre et libre choix. Quand un disciple était sous le Ndigueul, il ne fonctionnait que par lui ${ }^{(4)}$."Ainsi, le disciple se prépare en toute conscience à se donner entièrement à son engagement. Laccomplissement du Ndigueul devient la conséquence même de sa quête spirituelle et sa finalité : atteindre la pleine disponibilité d'un être libre et fidèle aux siens. 


\section{La matière sablonneuse des chemins secrets}

Ken Bugul ne savait pas d'emblée que l'ordre donné par le Marabout de se rendre régulièrement chez lui allait constituer le cadre d'un cheminement spirituel. Elle était seulement disponible pour le faire, et très vite elle a découvert en la personne du Marabout cet interlocuteur qu'elle attendait. "Ce fut ainsi qu'avec lui, je repris le chemin du purgatoire. [... Je n'avais jamais espéré rencontrer en ce monde quelqu'un avec qui je pouvais autant communiquer. Il me donna confiance en lui, en moi-même, en Dieu, en l'univers ${ }^{(42)}$." Sa vie est désormais rythmée par ses aller-retour le long du chemin de sable qui s'étire entre leurs deux concessions. Dans Riwan..., ce chemin de sable, auquel la narratrice prête une fonction purificatrice, symbolise une sorte de liant ontologique de la personne en quête d'unité qu'est Ken Bugul. En lisant le titre Riwan ou le chemin de sable, il apparaît que la disjonction n'est pas exclusive mais inclusive: Riwan est cet homme arrivé fou que le Marabout soigne par le Ndigueul en donnant un nouveau cadre à son existence. Tout comme la narratrice qui arpente ce sable, silencieux témoin et matière même du chemin qui la conduit vers elle-même.

Le Marabout représente le lien qui ramène Ken Bugul à ses racines familiales. Il est le détour salutaire par lequel sont passées les véritables retrouvailles avec la mère : "En arrivant au village, je ne pensais pas retrouver le Marabout, je pensais retrouver la Mère. Il me permit de la retrouver, de la découvrir, de l'aimer, de la reconnaître ${ }^{(43)}$." Restait à Ken Bugul à reprendre sa place parmi les autres. Or elle ne se savait pas non plus attendue à ce point. Son absence représentait un vide pour la société tout entière. "Le Serigne m'avait permis de retrouver ma place, cette place que personne ne pouvait occuper, cette place vide au milieu des miens, au centre de mon existence. Pour ma mère aussi cétait important. Cette réhabilitation, ma réhabilitation, était aussi la sienne ${ }^{(4)}$." Sous l'ceil de la société, les destins de la fille et de la mère sont liés. La fidélité de Ken Bugul à ses origines était (re)mise en question par son départ. Son retour a constitué une réponse qui se pose sous la forme d'une promesse : celle de la sauvegarde de cette identité collective qui s'exprime à travers elle, celle du maintien des autres à travers soi, de leur mémoire, de leur culture. "Ainsi le Serigne m'avait offert et donné la possibilité de me réconcilier avec moimême, avec mon milieu, avec mes origines, avec mes sources, avec mon monde, sans lesquels je ne pourrais jamais survivre.J'avais échappé à la mort de mon moi, de ce moi qui n'était pas à moi toute seule. De ce moi qui appartenait aussi aux miens, à ma race, à mon peuple, à mon village et à mon continent. Le moi de mon identitét ${ }^{(45)}$." Le parcours de Ken Bugul pourrait bien se lire comme la promesse tenue de la réconciliation qui seule peut assurer la permanence d'une identité morcelée par les clivages de l'histoire, les blessures de la vie et la violence du voyage entre deux mondes. 


\section{Notes}

1. Paul Ricceur, Soi-même comme un autre, Paris, Seuil, coll. Points, 1990, p. 139.

2. Ken Bugul, Le Baobab fou, Dakar, Les Nouvelles éditions africaines, 1996, p. 114. Paru pour la première fois en 1983, ce livre a depuis été réédité chez Présence africaine (Paris, 2010).

3. Ibid.

4. Ken Bugul, Le Baobab fou, op. cit., p. 132.

5. Ken Bugul, Le Baobab fou, op. cit., p. 33.

6. Ken Bugul, Riwan ou le chemin de sable, Paris, Présence africaine, 1999, p. 39.

7. Ken Bugul, Cendres et Braises, Paris, L'Harmattan, 1994, p. 143.

8. Frantz Fanon, Peau noire, masques blancs, Paris, Seuil, 1971, [1952], p. 149.

9. Ibid., p. 178.

10. Bernard Mouralis, Littérature et développement, Paris, éditions Silex, 1984, p. 54.

11. Lamine Diakhité, Présence africaine, $\mathrm{n}^{\circ} 56$, cité par B. Mouralis, op. cit., p. 42.

12. Ken Bugul, Le Baobab fou, op. cit., p. 67.

13. Ken Bugul, Le Baobab fou, op. cit., p. 101.

14. Ken Bugul, Le Baobab fou, op. cit., p. 102.

15. Ken Bugul, Le Baobab fou, op. cit., p. 74.

16. Ken Bugul, Le Baobab fou, op. cit., p. 98.

17. Ken Bugul, Le Baobab fou, op. cit., p. 107.

18. Ken Bugul, Le Baobab fou, op. cit., p. 102.

19. Ken Bugul, Le Baobab fou, op. cit., p. 123.

20. Ken Bugul, Le Baobab fou, op. cit., p. 181.

21. Ibid.

22. Ken Bugul, Cendres et Braises, op. cit., p. 119.

23. Ken Bugul, Cendres et Braises, op. cit., p. 118.

24. Ken Bugul, Cendres et Braises, op. cit., p. 133.

25. Ken Bugul, Cendres et Braises, op. cit., p. 133.

26. Ken Bugul, Cendres et Braises, op. cit., p. 103.

27. Ken Bugul, Cendres et Braises, op. cit., p. 56.

28. Ken Bugul, Cendres et Braises, op. cit., p. 103.

29. Ibid.

30. Ken Bugul, Cendres et Braises, op. cit., p. 33.

31. Ken Bugul, Cendres et Braises, op. cit., p. 22.

32. Ken Bugul, Cendres et Braises, op. cit., p. 40.

33. Ken Bugul, Riwan ou le chemin de sable, op. cit., p. 112.

34. Paul Ricoeur, Soi-même comme un autre, op. cit., p. 149.

35. Ken Bugul, Riwan ou le chemin de sable, op. cit., p. 111.

36. Paul Ricoeur, Soi-même comme un autre, op. cit., p. 311.

37. Paul Ricoeur, "Entre ontologie et éthique : la disponibilité", in Lectures, t. 2, Paris, Seuil, 1992,

1989 pour l'article, p. 75.

38. Ken Bugul, Le Baobab fou, op. cit., p. 179.

39. Ken Bugul, Riwan ou le chemin de sable, op. cit., p. 145.

40. Ken Bugul, Riwan ou le chemin de sable, op.cit., p. 181.

41. Ken Bugul, Riwan ou le chemin de sable, op. cit., p. 149.

42. Ken Bugul, Cendres et Braises, op. cit., p. 113.

43. Ibid.

44. Ken Bugul, Riwan ou le chemin de sable, op. cit., p. 168.

45. Ken Bugul, Riwan ou le chemin de sable, op. cit., p. 167. 